\title{
NEONATAL SEPTIC VASCULITIS: THREE CASES
}

\author{
F. Bennaoui1,2, N , El Idrissi Slitine 1,2, F.M.R Maoulainine 1,2 \\ 1. Neonatal Intensive Care Department, Mohammed VI University Hospital \\ 2. Research Team for Childhood, Health and Development, Marrakech School of Medicine, Cadi Ayyad University, Marrakech.
}

\section{INTRODUCTION}

Cutaneous vasculitis refers to a large and heterogeneous group of diseases, which have in common inflammatory involvement of the wall of arterioles, venules or capillaries of the dermis.

Septic vasculitis is a rare and serious condition in the newborn that can complicate critical sepsis.

\section{OBSERVATIONS}

We report three observations of male newborns admitted to

neonatal unit for septic vasculitis complicated with severe sepsis.

\section{Observation 1:}

A 13-day newborn who had a history of consanguinity with two neonatal deaths including one in the same table, was admitted for extensive necrotic lesions appeared at the 10th day of life at the foot and the scalp with an unencrypted fever.

On clinical examination : in addition to necrosis lesions a cutaneo-mucous pallor.

Biological assessment revealed anemia, hyperleukocytosis with very high CRP, lumbar puncture and urine culture were sterile.

The treatment consisted, in a C3GGentamicin with corticosteroid therapy.

The outcome The evolution was fatal, the newborn died the day after his hospitalization.

\section{Observation 2:}

A 12-day old newborn with no particular pathological history. The infectious anamnesis was positive. The newborn was admitted for perinatal asphyxia with sepsis

On clinical examination had a septic complexion, dehydration Table $\mathrm{B}$, hypotonia with weak reflexes, patches of necrosis in the feet and large toes rapidly progressive (Figure I).

Biological assessment revealed anemia, leuko-neutropenia, thrombocytopenia with CRP elevated to $93 \mathrm{mg} / \mathrm{I}$, skin biopsy was not performed.

The treatment consisted of bi-antibiotic treatment with C3G-Gentamicin and then ciprofloxacin. The newborn died at J22 of life in a septic shock chart.

\section{Observation 3:}

It was a newborn child of 4 days, he had a history of death born in the siblings. The infectious anamnesis was negative. The newborn was admitted for infectious jaundice and bullous and necrotic lesions appeared at 1 day of life (Figure II).

On clinical examination the newborn was jaundiced with bleeding blebs and rapidly extensive plaques of necrosis (Figure III).

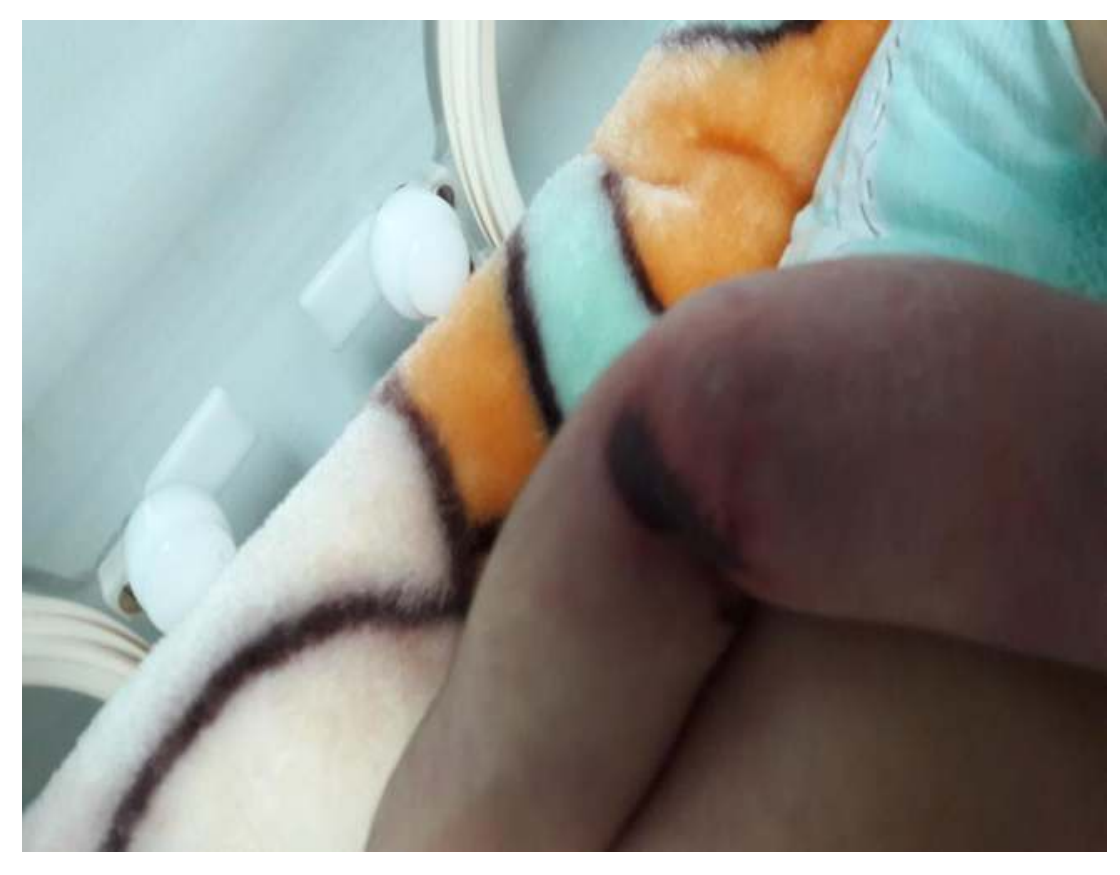

Biological assessment found thrombocytopenia with elevated CRP $37.54 \mathrm{mg} / \mathrm{l}$, staphylococcal coagulase negative blood culture, and cutaneous biopsy showed coagulase-negative staphylococcus.

The treatment consisted of bi-antibiotic therapy based on C3G-Gentamicin in addition to sepsis management. The newborn died at $\mathrm{J} 8$ of life in a septic shock chart

\section{DISSCUSION}

The cutaneous vasculitis can be generalized or localized, the diameter of the vessels is variable ranging from capillaries of the papillary dermis to arterioles and venules of the deep dermis or subcutaneous tissue, frequent presence of an associated purpura.

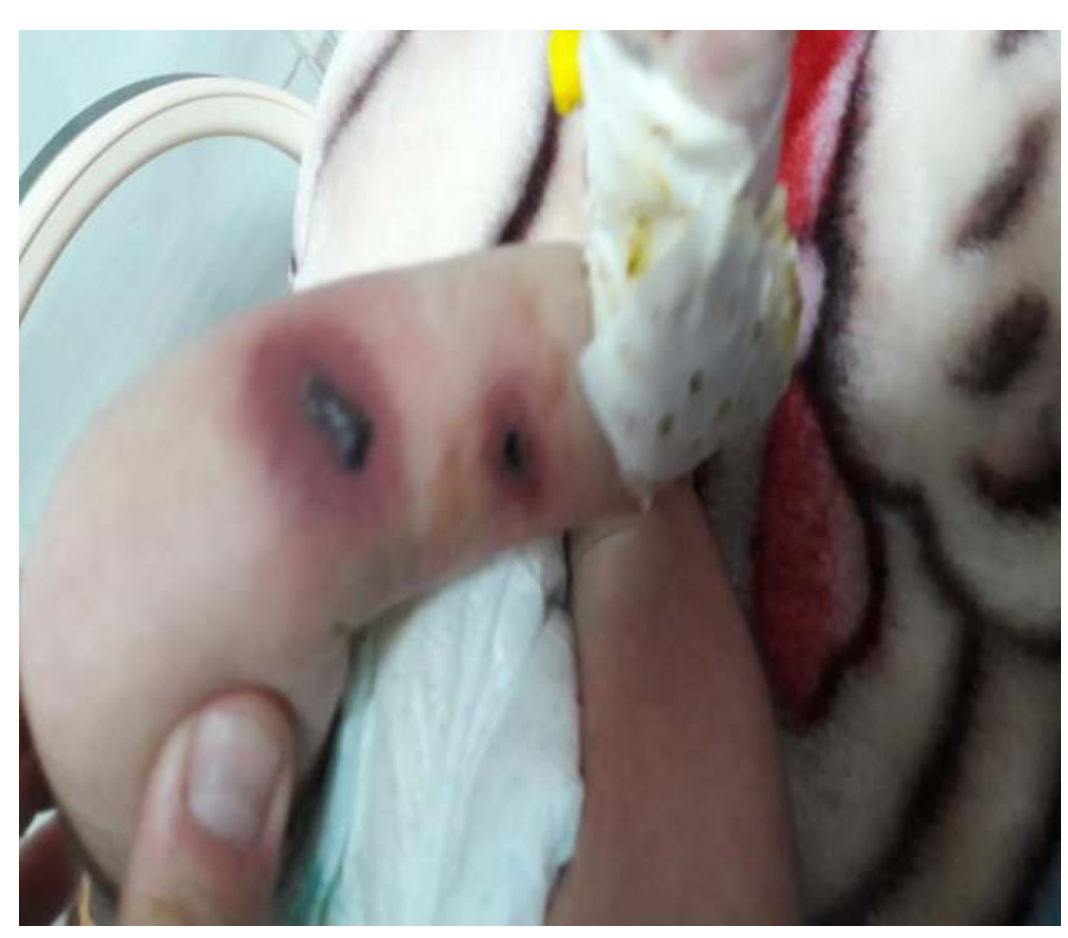

The clinical aspects are varied; with often several types of elementary lesions associated giving a polymorphous appearance

La vasculite cutanée peut être idiopathique (45$55 \%$ ), infectieuse (15-20\%) (atteintes bactériennes, virales, parasitaires ou fungiques), associée à des maladies inflammatoires $(15-20 \%)$, à la prise de médicament (10-24\%) ou à des néoplasies (2-5\%) .

Confirmation of vasculitis in front of purpuric lesions is valuable because there are many other causes of purpura: after removing a hematologic cause (thrombocytopenia), the cause of a purpura is certainly vascular. It can be either a vasculopathy or a vasculitis.

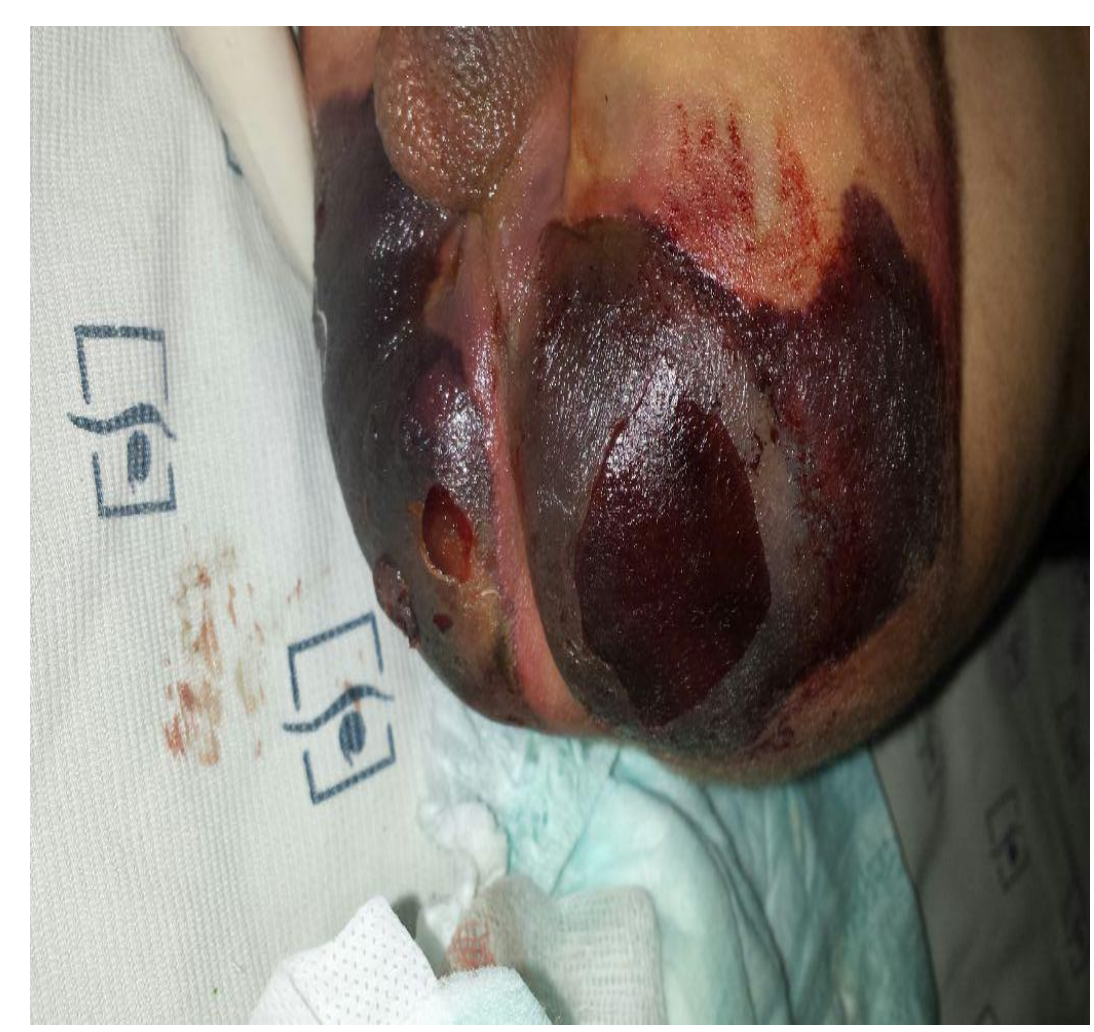

These vasculitis are linked to the proliferation of certain organisms

infectious in the endothelium or vascular wall, resulting in occlusion and then necrosis.

The microorganisms responsible for these occlusions are (not

exhaustive) bacteria: Klebsiella pneumoniae, Morganella Morganii, Pseudomonas aeruginosa, Serratia marcesens, Vibrio vulnificus, or fungi: Absidia, Aspergillus fumigatus, Candida albicans, Fusarium, Mucor, Rhizopus

These tables are mainly found in immunocompromised patients,

including neutropenic and the newborn.

The prognosis is related to the early diagnosis and the implementation of a suitable anti-infective treatment. Most of the time the prognosis is reserved for the newborn; caused by the rapid installation of septic shock.

\section{CONCLUSION}

Septic vasculitis at birth or the neonatal period is a rare clinical problem, often complicating sepsis that is not or poorly controlled.

In such a context, a cutaneous involvement has several interests: it represents an easy access to the clinician's view and the biopsy, and thus allows to confirm the diagnosis of vasculitis. 